\title{
Efficient Ultrashort Pulsed Laser Processing by Dynamic Spatial Light Modulator Beam Shaping for Industrial Use
}

\author{
Christian Lutz ${ }^{* 1}$, Gian-Luca Roth ${ }^{1}$, Stefan Rung ${ }^{1}$, Cemal Esen ${ }^{2}$, and Ralf Hellmann ${ }^{1}$ \\ ${ }^{1}$ Applied Laser and Photonics Group, \\ University of Applied Science Aschaffenburg, \\ Wuerzburger Strasse 45, 63743 Aschaffenburg, Germany \\ ${ }^{2}$ Applied Laser Technologies, \\ Ruhr University Bochum, \\ Universitaetsstrasse 150, 44801 Bochum, Germany \\ *Corresponding author's e-mail: Christian.Lutz@th-ab.de
}

\begin{abstract}
We report on the effect of different transversal beam shapes on the efficiency of ablation processes and the resulting surface characteristics. A possibility to efficiently apply ultrashort pulsed lasers with high average power is beam shaping. By using a cooled reflective based liquid crystal spatial light modulator to generate different spot distributions, it is possible to spatially allocate the available power to avoid excessive high fluences. In our experiments, we determine the optimal fluence to ablate the maximum volume per watt to be in the range of $0.2-0.4 \mathrm{~J} / \mathrm{cm}^{2}$. Based on this fluence, we increase the number of spots from one to a maximum of twenty to ablate steel in a multilayer scanning-based process. In this context, we examine the influence of different separation distances between the spots on the ablation efficiency and roughness. Subsequently to these investigations, we develop an efficient roughing process with higher ablation rates and a nearly constant roughness.
\end{abstract}

DOI: 10.2961/jlmn.2021.01.2011

Keywords: laser material processing, beam shaping, ultrashort pulsed laser, ablation rate, ablation efficiency, spatial light modulator

\section{Introduction}

The average power of industrial ultrashort pulsed lasers has been significantly increased up to several hundreds of Watts over the last years [1; 2]. However, those high average powers are not suitable for common ablation processes as resulting fluences are exceeding the efficient process regime and may deteriorate ablation quality. A reasonable fluence regime for efficient ablation of e.g. steel has been determined to $0.2-0.4 \mathrm{~J} / \mathrm{cm}^{2}$ [3; 4]. Thus, the used average power for a single $41 \mu \mathrm{m}$ spot, as used in our experimental study, is only around $1 \mathrm{~W}$ at a laser pulse repetition rate of $200 \mathrm{kHz}$. A higher laser power results in negative effects like heat accumulation [5; 6], plasma shielding [7] and as a consequence to a lower ablation efficiency [6].

Therefore, there is a high demand on new micromachining strategies, especially for large area applications [8; 9]. A possibility to apply a high average laser power in an efficient micromachining process is by using a high laser pulse repetition rate, which usually challenges conventional scanning system like galvanometer scanners. Ultrafast deflection systems such as polygon scanners or acusto-optic deflectors are capable to deliver the required deflection speed. They separate single laser pulses at high repetition rate at the cost of a more complex setup and reduced accuracies. Another approach to distribute the available laser power is by using spatial beam shaping technology [10; 11]. Optical beam shaping can be achieved by different solutions like digital mirror devices, deformable mirrors, diffractive optical elements (DOE), acusto-optic deflectors and spatial light modulators (SLM) [12]. With these optical elements it is possible to shape the beam towards the desired spatial intensity distribution including multi-beam arrays, top-hat profiles and Bessel beams [13-15]. Especially DOEs are widely used for creating multi spot arrays for efficient parallelization of micromachining processes as they can withstand the intensities of high power ultrashort pulse lasers [16; 17]. While DOEs are fixed to one beam profile, SLMs represent a dynamic way to create process adapted beam profiles. During the last decade, research topics on SLMs were focused on microscopy imaging [18-20] and internal micro processing of transparent materials [21-23], applications which do not demand for a high optical power resistance.

Micromachining using SLM devices has also been demonstrated for lower power solution, including drilling and marking applications using a parallel processing setup [24]. By using a high reflective, actively cooled SLM, this technology is nowadays also accessible for high power scanning ablation processes, which can profit from application adapted spot patterns. In combination with a commonly used galvanometer scanner, this method allows a customization of the spot formation on the respective micromachining process [8]. In this study, we qualify an efficient ultrashort pulse laser micromachining process by using an adaptive SLM beam splitting approach. The influence of generated spot number, their spatial distribution and respective spatial separation on the scanning ablation process is characterized by the resulting ablation rate, ablation efficiency and achievable roughness, respectively. 


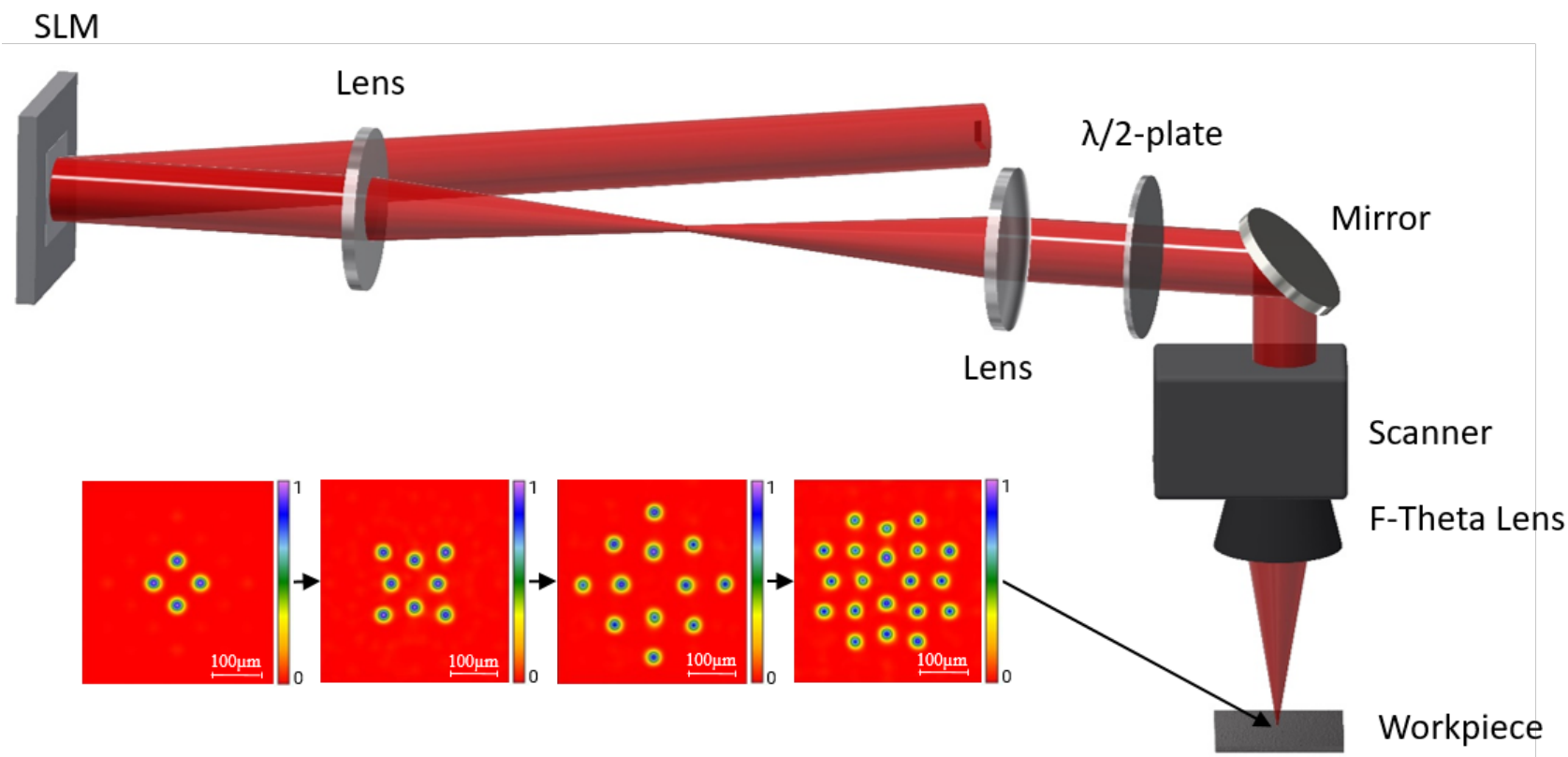

Fig. 1 Schematic illustration of the setup for femtosecond laser ablation using a spatial light modulator (SLM) and a 4f telescope with a galvo scanner including an F-Theta lens. Also shown are simulated intensity profiles for four-, eight-, twelve- and twentyfold spot distributions (Lighttrans Virtual Lab).

\section{Experimental}

We use an Yb:YAG laser (Amplitude Tangor) with a wavelength of $1030 \mathrm{~nm}$ and a maximum power of $100 \mathrm{~W}$. The laser is integrated into a micromachining system (Pulsar Photonics, RDX-1000), which uses a galvo scanner (Scanlab, IntelliSCAN $\mathrm{se}_{\mathrm{s}} 14$ ) and a F-Theta lens (QiOptiq, LINOS FTheta Ronar) with a focal length of $100 \mathrm{~mm}$. The focal diameter $d_{o}$, measured by a high-resolution CCD camera (IDS, UI149xLE) is $41 \mu \mathrm{m}\left(1 / e^{2}\right)$. The respective fluence is calculated by $\Phi=E_{p} /\left(\pi \cdot r_{0}^{2}\right)$, with $E_{p}$ being the pulse energy and $r_{0}$ the radius of the focal beam.

Experiments are performed using a pulse repetition rate of $200 \mathrm{kHz}$ with 800 fs pulse duration (FWHM, measured after

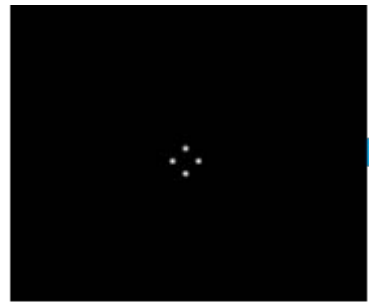

a)

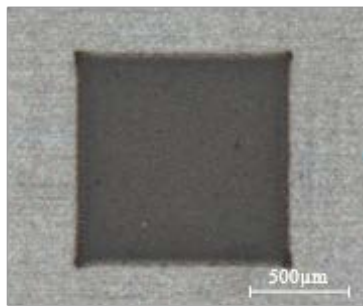

d)

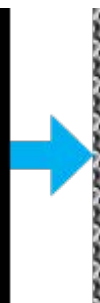

b)

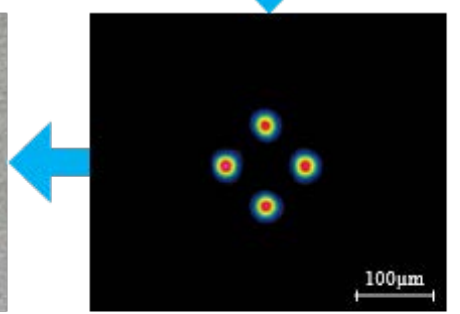

c)
Fig. 2 a) Target file of the spot distribution with a separation of $100 \mu \mathrm{m}$ b) IFTA calculated CGH for the mentioned beam profile c) Focus with four spots with a separation of $100 \mu \mathrm{m}$ and a uniformity of 0.96 d) Hatched stainless steel sample with $100 \mu \mathrm{m}$. laser aperture by an autocorrelator) and a scanning speed of $2968 \mathrm{~mm} / \mathrm{s}$, resulting in a pulse overlap of $64 \%$. The SLM based beam shaping module (cf. Fig.1) allows dynamic beam shaping to generate different spot distributions with a maximum frame rate of $60 \mathrm{~Hz}$. To protect the LCOS-SLM (Hamamatsu X15223), an effective active liquid cooling system is integrated. Required computer generated (CGH) holograms are calculated using an iterative flourier transform algorithm. The workflow for generating the different spot distributions is exemplarily shown in Fig. 2. After the initial calculation of the computer generated hologram, the uniformity of the single sub-beams in the focal plane is further improved by a feedback loop based weighted Gerchberg-Saxton algorithm [25]. As the diffraction efficiency for each CGH differs, we control the total reflective power from the SLM using a power meter (Gentec EO, UP25N 100H H9) to ensure equal power impingement for each beam profile. The ultrashort pulse laser is used to ablate a defined geometry i.e. a cavity in a precise layer-by-layer ablation process by scanning the laser spot over the sample. The bidirectional hatched area has a size of $2 \times 2 \mathrm{~mm}^{2}$. By adding an additional rotation of $\alpha=11^{\circ}$ after each hatch, we obtained a homogenous irradiated surface after 50 scans. The ablated stainless steel (X5CrNi18-10) structures are analyzed by a laser-scanning microscope (Keyence, VK-X200series) and a scanning electron microscope (Tescan, Maia3 TriglarTM).

\section{Results and discussion}

\subsection{Ablation rate and efficiency}

Ablation characteristics are studied by employing different laser powers and two different beam profiles, a single spot and a three-spot distribution with a distance of $72 \mu \mathrm{m}$ between the individual spots. The laser power range for the three-spot geometries is tripled to retain the same fluence per spot. The influence of the laser power on the ablation effi- 


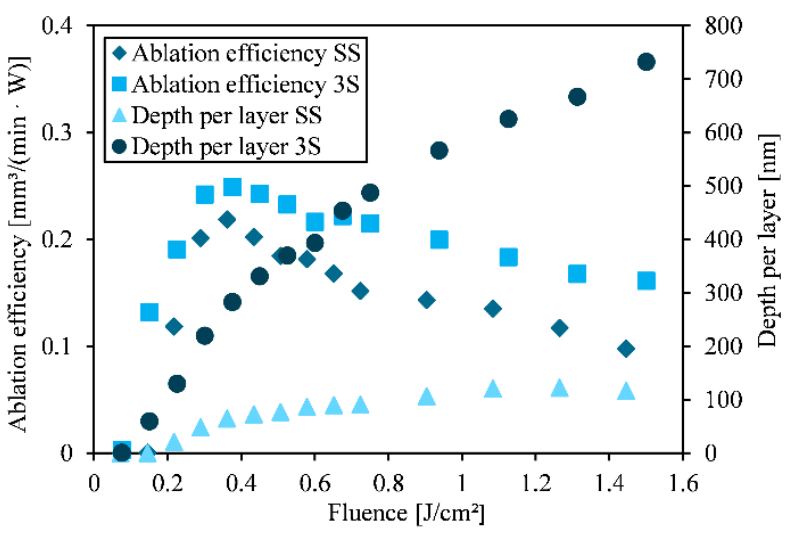

Fig. 4 Ablation efficiency and depth per layer for fluencies between 0.1 and $1.6 \mathrm{~J} / \mathrm{cm}^{2}$. The ablation efficiencies are calculated of the middle level of basic surface and ablated surface, the calculated process time and the used laser power. The depth per layer is the average of the scanned 50 layers.

ciency is depicted in Fig. 3. Both, single- and multi-spot profiles show similar trends of the ablation rate including a prime fluency range between 0.3 and $0.5 \mathrm{~J} / \mathrm{cm}^{2}$.

These findings are in accordance to previous studies by Hodgson et al., showing an optimal fluence between 0.3$0.4 \mathrm{~J} / \mathrm{cm}^{2}$ for a pulse overlap of $60 \%$ [4] and by Neuenschwander et al., reaching an ablation efficiency of around $0.25 \mathrm{~mm}^{3} /(\mathrm{min} \cdot \mathrm{W})$ for stainless steel [3; 26]. In addition, we find that by using the optimal fluence a tripled ablation depth per layer can be achieved by using a three-spot profile. For the maximum fluence of $1.5 \mathrm{~J} / \mathrm{cm}^{2}$, the ablated depth per layer is about seven times higher.

The influence of different spot separation distances in a multi-spot array is studied by increasing the distance stepwise between 72 and $570 \mu \mathrm{m}$ using a three-spot-profile. The corresponding beam profile is illustrated in Fig. 4 with a separation of $125 \mu \mathrm{m}$. In addition, the normalized intensity profile in $\mathrm{x}$ and $\mathrm{y}$ direction of each spot is given. The specified roughness $R_{a}$, calculated from an $80 \times 60 \mu m^{2}$ rectangular, of the ablated surface is given in Fig. 5 for different

fluencies. Obviously, higher laser fluence results in larger roughness, a typical effect in laser ablation processes. In the

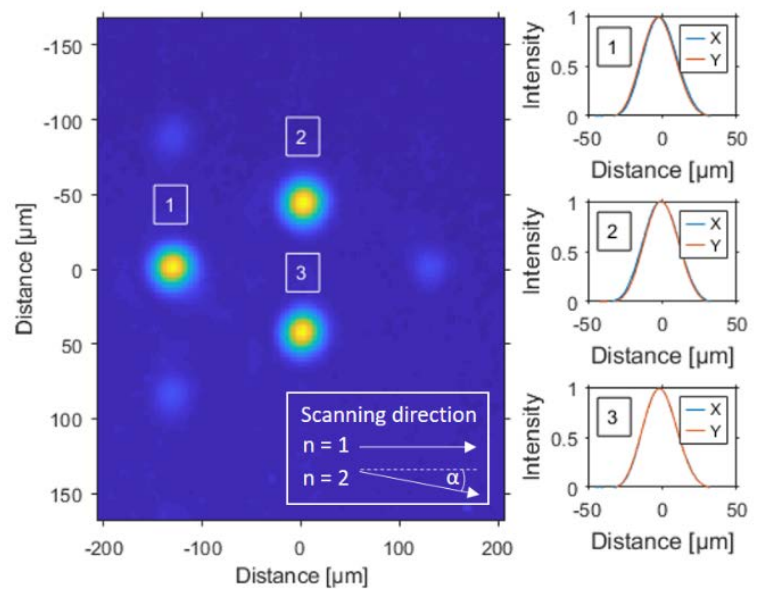

Fig. 3 Tree-spot-profile with a separation of $125 \mu \mathrm{m}$, measured with a spot monitor at the focus plane and the normalized intensity profiles in $\mathrm{x}$ and $\mathrm{y}$ direction for each spot. The rotation angel $\alpha$ amounts $11^{\circ}$ per layer.

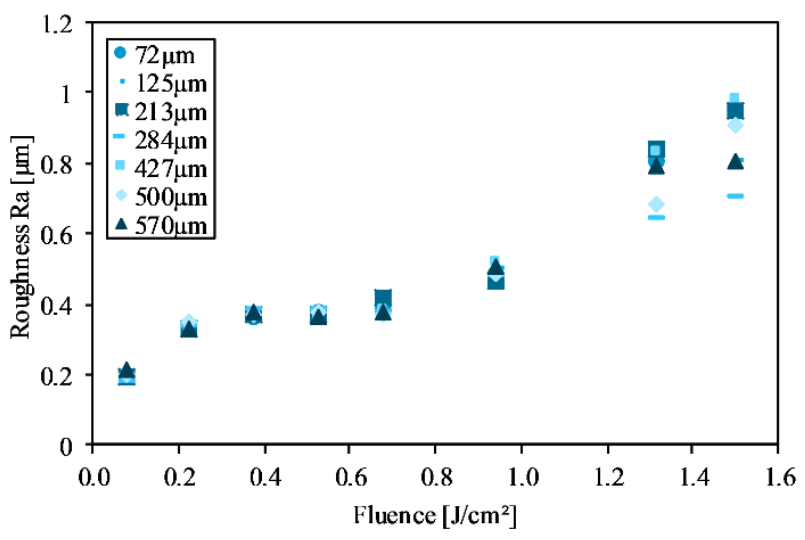

Fig. 5 Effect on the roughness of different spot separations and fluencies between 0.1 and $1.6 \mathrm{~J} / \mathrm{cm}^{2}$. The measured surface is a rectangle of $80 \times 60 \mu \mathrm{m}$ on the bottom with 50 layers ablated volume, recorded with a $150 \mathrm{x}$ enlargement.

studied range, roughness $R_{a}$ between 0.2 and $1.0 \mu \mathrm{m}$ are achieved, which is comparable to previous studies on laser ablation of metal surfaces [27; 28]. In particular, Wu et al. reach a roughness around $1 \mu \mathrm{m}$ for a single spot process with 60 layers and $0.25-0.35 \mathrm{~J} / \mathrm{cm}^{2}$ [29]. We also find that different spot separations have an insignificant influence on the resulting roughness in the range between $0.1-1.0 \mathrm{~J} / \mathrm{cm}^{2}$. In addition, the ablation rate remains at a constant level of $0.25 \mathrm{~mm}^{3} /(\mathrm{W} \cdot \mathrm{min})$. As both, ablation rate and roughness do not depend on spot separation distance in a three-spot beam profile, we as a beneficial consequence gain a high flexibility for further spot distributions.

Based on these preliminary results on the ablation efficiency, we study the influence of different spot distributions with different numbers of sub-beams in a fluence range between 0.2 and $0.8 \mathrm{~J} / \mathrm{cm}^{2}$. Due to the low fluence required in each spot, a higher number of sub-beams can be implemented in our spot distributions to further increase the ablate rate. To achieve a maximum fluence of $0.75 \mathrm{~J} / \mathrm{cm}^{2}$, using a $41 \mu \mathrm{m}$ spot, a laser power around $2 \mathrm{~W}$ is needed in each spot. Limited by the SLM, the maximum usable power in our experimental setup is around $45 \mathrm{~W}$. The number of sub-beams with a constant separation of $100 \mu \mathrm{m}$ is stepwise increased from 1 to 20. The ablation rates for these spot distributions

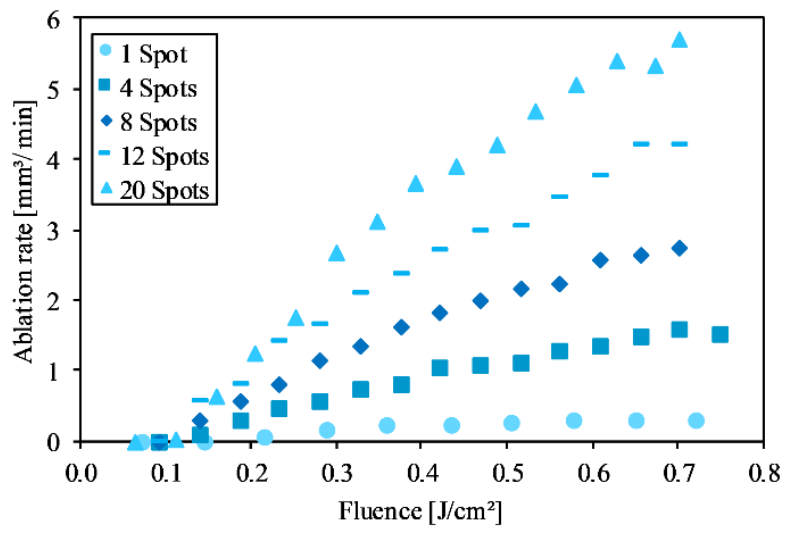

Fig. 6 Effect of 1, 4, 8, 12 and 20 spots with a separation of 100 $\mu \mathrm{m}$ and fluences between 0.1 and $0.8 \mathrm{~J} / \mathrm{cm}^{2}$ on the ablation rate. The shown ablation rate is the calculated average after 50 scanning layers. 


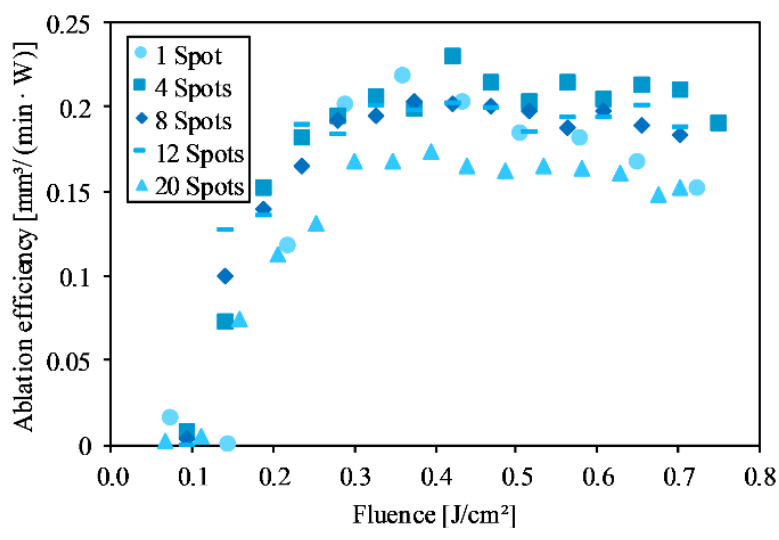

Fig. 7 Effect of 1, 4, 8, 12 and 20 spots and fluences between 0.1 and $0.8 \mathrm{~J} / \mathrm{cm}^{2}$ on the ablation efficiency. The ablation efficiencies are calculated of the middle level of basic surface and abated surface, the process time and the used laser power.

are given in Fig. 6 using different fluences. For each individual spot distribution, the ratio of raising fluence and ablation rate has a linear slope. Moreover, we see a correlation between the number of spots and the ablated volume. These characteristics promise an easy scalable process regarding the number of spots and the used process power.

In this experiment, a single spot has a maximum ablation rate of $0.3 \mathrm{~mm}^{3} / \mathrm{min}$ at a fluence of $0.75 \mathrm{~J} / \mathrm{cm}^{2}$. The four and eight spot distribution reach a rate of 1.5 and $2.75 \mathrm{~mm}^{3} / \mathrm{min}$, respectively, i.e. by quadrupling the laser power and distributing on four spots, a five times higher ablation rate is achieved, which corresponds to a factor of 1.25 per spot. This correlation is also observable for the eight and twelve spot distribution. While the overall increase of the ablation rate with the number of spots can be attributed to the increasing average power that is applied to the work piece (please bear in mind that the individual fluence per spot is set to be constant), the observed surplus in ablation rate is assigned to heating effects at high repetition rates as we turn the ablation scan by $11^{\circ}$ after each hatch. For twenty spots, however, this general trend can not be approved and we find an ablation rate of $5.7 \mathrm{~mm}^{3} / \mathrm{min}$, corresponding to a factor of only 0.95 as compared to the single spot. This can be attributed to a decreasing diffraction efficiency of the CGH at a higher number of spots. The amount of unshaped laser power between the spots gets larger, but the fluence is not high enough to remove any material. Another reason is a reduced uniformity between the single spots resulting in differences of the fluence in each spot. Thus, the ablation efficiency for a 20 spot pattern is lower as compared to a smaller number of spots as shown in Fig. 7. By using up to 12 spots, the maximum ablation efficiency remains at a constant level of around $0.2 \mathrm{~mm}^{3} /(\mathrm{min} \cdot \mathrm{W})$. Nevertheless, the ablated volume can be increased by using a larger number of spots with constant fluence as depicted in Fig. 6.The ablation efficiency is similar to a previous study using a similar laser setup [30].

\subsection{Roughness for different spot distributions}

Next to process efficiency, laser ablation is usually characterized by the roughness of the ablated surface. In this study, the roughness is measured inside an $80 \times 60 \mu^{2}$ rectangle by determining and averaging the profile surface parameter Ra along multiple lines set within this area. As the

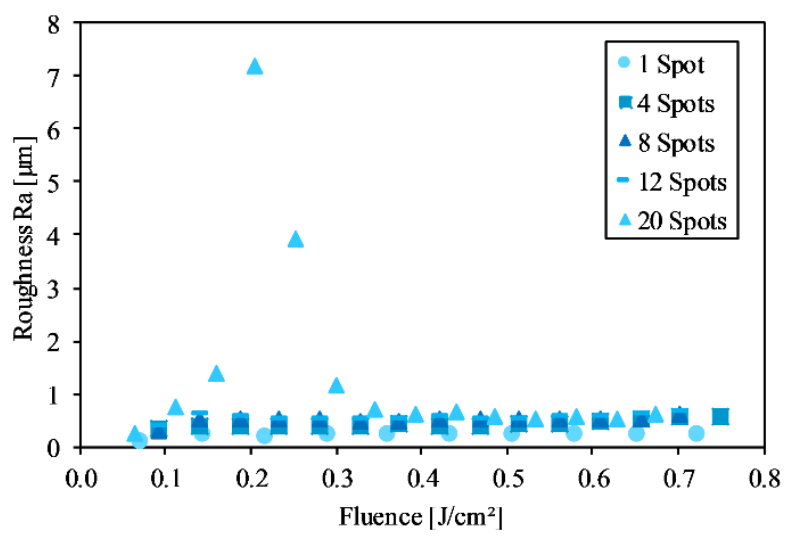

Fig. 8 Surface roughness Ra of different spot distributions and fluencies between 0.1 and $0.8 \mathrm{~J} / \mathrm{cm}^{2}$. The measured surface is a rectangle of $80 \times 60 \mu \mathrm{m} 2$ on the bottom of the 50 layers ablated volume, recorded with a $150 \mathrm{x}$ enlargement.

roughness parameter $R_{a}$ is the arithmetic mean of all profile values, we also evaluate the more informative parameter $R_{z}$. Basically both, $R_{a}$ and $R_{z}$ roughness show a qualitative similar slope. The initial roughness of the basic material is around $R_{a}=0.16 \mu \mathrm{m}$ and $R_{a}=3.44 \mu \mathrm{m}$. The roughness of the ablated surface is shown in Fig. 8 using $R_{a}$ for the different spot distributions and fluences. Aside of one peak at a fluence of $0.25 \mathrm{~J} / \mathrm{cm}^{2}$, we see a constant roughness of below $\mathrm{R}_{\mathrm{a}}=0.25 \mu \mathrm{m}, \mathrm{R}_{\mathrm{z}}=6.3 \mu \mathrm{m}$ being is typical for the studied fluence range [27-29]. The lowest roughness is achieved by using a single spot ablation process. By using a higher number of spots, the roughness slightly increases. The minimum of $\mathrm{R}_{\mathrm{a}}=0.14 \mu \mathrm{m}, \mathrm{R}_{\mathrm{z}}=5.16 \mu \mathrm{m}$ shows a polishing like process for a low fluence of $0.75 \mathrm{~J} / \mathrm{cm}^{2}$. Wu et al. measured a roughness of approximately $0.4 \mu \mathrm{m}$ after 40 ablated layers [29]. With a lower repetition rate of $1 \mathrm{kHz}$, Cheng et al. produced similar surfaces on other metals around a $R_{a}$ value of $0.2 \mu \mathrm{m}$ [28]. Generally, we only see a small effect on roughness $R_{a}$ and $\mathrm{R}_{\mathrm{z}}$ for different number of spots. Nevertheless, a finishing process with a low fluence can be applied to minimize the roughness and increase the surface quality.

For high spot numbers and at a laser fluence of $0.25 \mathrm{~J} / \mathrm{cm}^{2}$, i.e. high applied average laser power, we however, find a maximum roughness of $\mathrm{Ra}=7 \mu \mathrm{m}(\mathrm{Rz}=40 \mu \mathrm{m})$, which results from the formation of cone shaped microstructures with a height of up to $30 \mu \mathrm{m}$ and diameters in the range between 10 and $40 \mu \mathrm{m}$. As it has previously been reported in similar process parameter regimes for multilayer laser ablation, different microstructures like laser induced periodic surface structures, cones from impurities and cone-like protrusions may appear on stainless steel, depending on the applied fluence, the accumulated energy and the number of passes [31; 32]. Previous hypotheses assert that molten and solidified ablation particles or oxides formed the individual cones [33]. The appearance of cones is illustrated in figure 9, showing SEM images of the surface for a different number of spots. Here the fluence per spot is constantly $0.25 \mathrm{~J} / \mathrm{cm}^{2}$, i.e. with increasing spot number the applied average power increases. Obviously, laser induced periodic surface structures (LIPSS) are found on each ablated surface, while the evolution of cones starts at surfaces ablated by using 12 and 20 spots. Dark areas at the cone summits indicate inclusion induced cones [31]. In addition to that, inclusion induced 

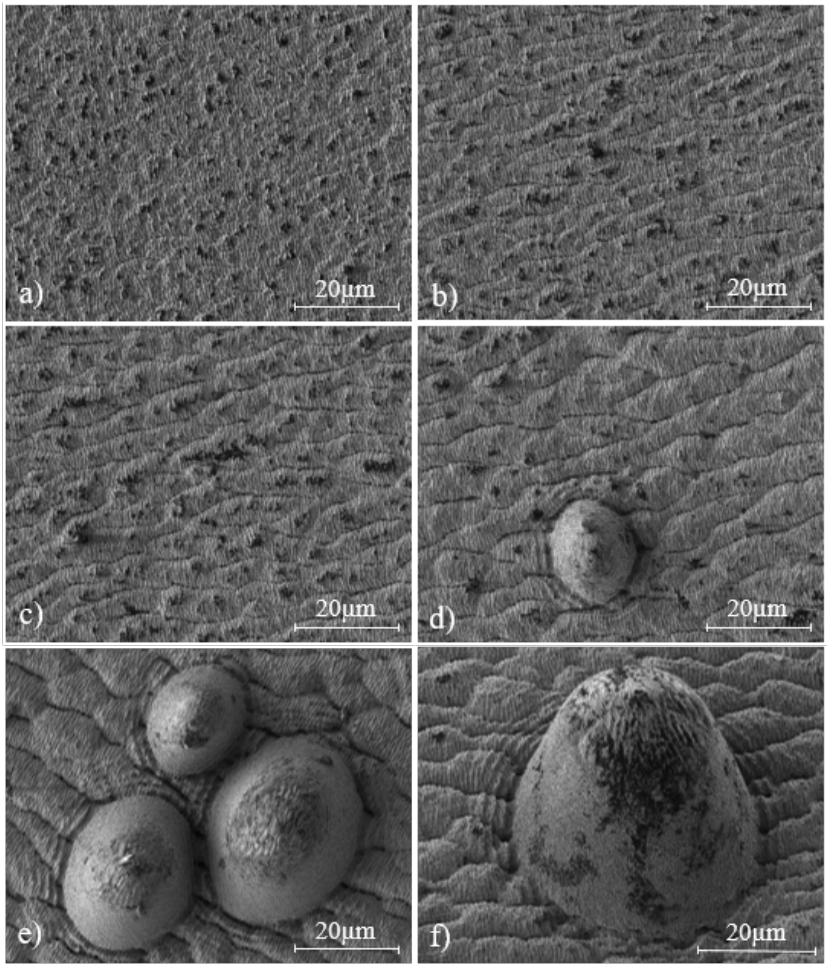

Fig. 9 Illustrations of the different surfaces after 50 layers ablation, taken with a scanning electron microscope, a) 1 Spot $0.8 \mathrm{~W}$, b) 4 Spots $3 \mathrm{~W}$, c) 8 Spots $6 \mathrm{~W}$, d) 12 Spots $9 \mathrm{~W}$, e) 20 Spots $13.5 \mathrm{~W}$, f) 20 Spots $13.5 \mathrm{~W}$, under a $40^{\circ}$ angel.

cones are also created using a smaller spot distribution, but lower in number and less pronounced not being representative in describing the ablated surface. We assign the increasing number of micro-cones for higher spot numbers to the higher average laser power that is required as to guarantee a constant laser fluence in each individual spot. This higher average laser power in turn leads to a higher resulting process temperature, advancing the cone generation.

\section{Conclusion}

We demonstrate a highly efficient femtosecond laser ablation process of stainless steel for different laser spot distributions, based on beam shaping by a spatial light modulator. To use the available ultrashort pulsed laser power in a highly efficient way, we employ a low fluence per spot and multispot beam profiles. To increase the ablation rate, we raise the number of spots up to 20, finding ablations rates of up to $6 \mathrm{~mm}^{3} / \mathrm{min}$ with an almost constant ablation efficiency of $0.2 \mathrm{~mm}^{3} /(\min \cdot \mathrm{W})$. By multiply the spot distributions, we find a correlation between the spot number and the ablation rate with a factor of 0.9 for the 20 spot profile and a factor of 1.25 for the lower spot numbers. In addition, we show that in a fluence range of up to $1.6 \mathrm{~J} / \mathrm{cm}^{2}$ varying spot separations between 70 and $570 \mu \mathrm{m}$ have negligible influences on the ablation rate and roughness. Compared to single spot processes, which result in a roughness of $R_{a}=0.25 \mu \mathrm{m}$, using multiple spots leads to slightly higher $\mathrm{R}_{\mathrm{a}}$ between 0.4 and $0.6 \mu \mathrm{m}$ unless micron-sized cones appear.

\section{References}

[1] P. Russbueldt, T. Mans, G. Rotarius, J. Weitenberg, H.D. Hoffmann, and R. Poprawe: Opt. Express, 17, (2009) 15.

[2] P. Russbueldt, T. Mans, J. Weitenberg, H. D.

Hoffmann, and R. Poprawe: Opt. Lett., 35, (2010) 24.

[3] B. Neuenschwander, B. Jaeggi, M. Schmid, and G. Hennig: Phys. Procedia, 56, (2014) 1047.

[4] N. Hodgson, S. Heming, A. Steinkopff, H. Haloui, and T. Lee: Proc. of Lasers in Manufacturing Conference 2019, (2019) 151.

[5] F. Bauer, A. Michalowski, T. Kiedrowski, and S. Nolte: Opt. Express, 23, (2015) 2.

[6] J. Finger and M. Reininghaus: Opt. Express, 22, (2014) 15.

[7] J. König, S. Nolte and A. Tünnermann: Opt. Express, 13, (2005) 26.

[8] A. Gillner, J. Finger, P. Gretzki, M. Niessen, Thilo Bartels, and M. Reininghaus: J. Laser Micro Nanoeng., (2019) 129.

[9] S. Bruening, K. Du, M. Jarczynski, G. Jenke, and A. Gillner: Procedia CIRP, 74, (2018) 254.

[10] O. Bernard, E. Audouard, B. Schöps, M. Delaigue, G. Dalla-Barba, K. Mishchik, C. Hönninger, and E. Mottay: Procedia CIRP, 74, (2018) 310.

[11] J. Schille, L. Schneider, S. Mauersberger, S. Szokup, S. Höhn, J. Pötschke, F. Reiß, E. Leidich, and U. Löschner: Lubricants, 8, (2020) 3.

[12] P. S. Salter and M. J. Booth: Light Sci Appl, 8, (2019) 10565.

[13] A. Hendriks, D. Naidoo, F. S. Roux, C. López-Mariscal, and A. Forbes: Proc. SPIE, Vol. 8492, (2012) 849006. [14] S. Orlov, V. Vosylius, P. Gotovski, A. Grabusovas, J. Baltrukonis, and T. Gertus: J. Laser Micro Nanoeng., (2018) 280.

[15] S. Schwarz, S. Rung, C. Esen, and R. Hellmann: J. Laser Micro Nanoeng., 13, (2018) 2.

[16] C. He, K. Vannahme, A. Gillner: J. Laser Micro Nanoeng., (2019) 95.

[17] C. He, M. Steger, and A. Gillner: J. Laser Micro Nanoeng., 13, (2018) 1.

[18] C. Maurer, A. Jesacher, S. Bernet, and M. RitschMarte: Laser \& Photon. Rev., 5, (2011) 1.

[19] P. Pozzi, D. Gandolfi, M. Tognolina, G. Chirico, J. Mapelli, and E. D'Angelo: Neurophotonics, 2, (2015) 1. [20] N. Matsumoto, T. Inoue, A. Matsumoto, and S. Okazaki: Biomed Opt Express, 6, (2015) 7.

[21] L. Huang, P. S. Salter, F. Payne, and M. J. Booth: Opt. Express, 24, (2016) 10.

[22] G.-L. Roth, S. Rung, C. Esen, and R. Hellmann: Opt. Express, 28, (2020) 4.

[23] P. S. Salter, M. J. Woolley, S. M. Morris, M. J. Booth, and J. A. J. Fells: Opt. Lett., 43, (2018) 24.

[24] Z. Kuang, W. Perrie, J. Leach, M. Sharp, S. P. Edwardson, M. Padgett, G. Dearden, and K. G. Watkins: Appl. Surf. Sci., 255, (2008) 5.

[25] P. Memmolo, L. Miccio, F. Merola, A. Paciello, V. Embrione, S. Fusco, P. Ferraro, and P. Antonio Netti: Opt. Lasers Eng., 52, (2014) 206. 
[26] B. Neuenschwander, B. Jaeggi, M. Schmid, V.

Rouffiange, and P.-E. Martin: Proc. SPIE, Vol. 8243 (2012) 824307.

[27] A. Žemaitis, M. Gaidys, P. Gečys, G. Račiukaitis, and M. Gedvilas: Opt. Lasers Eng., 114, (2019) 83.

[28] J. Cheng, W. Perrie, S. P. Edwardson, E. Fearon, G. Dearden, and K. G. Watkins: Appl. Surf. Sci., 256, (2009) 5.

[29] B. Wu, P. Liu, F. Zhang, J. Duan, X. Wang, and X. Zeng: Appl. Phys. A, 124, (2018) 1.

[30] G. Mincuzzi, A. Rebiere, J. Lopez, M. Faucon, and R. Kling: Proc. SPIE, Vol. 10520 (2018) 10520.

[31] V. Villerius, H. Kooiker, J. Post, and Y. T. Pei: Int. J. Mach. Tools Manuf., 138, (2019) 27.

[32] E. J. Y. Ling, J. Saïd, N. Brodusch, R. Gauvin, P. Servio, and A.-M. Kietzig: Appl. Surf. Sci., 353, (2015) 512.

[33] M. Tsukamoto, T. Kayahara, H. Nakano, M. Hashida, M. Katto, M. Fujita, M. Tanaka, and N. Abe: Opt. Commun., 59, (2007) 666.

(Received: June 26, 2020, Accepted: February 23, 2021) 